\title{
Facial nerve paralysis due to intra aural tick infestation: a case report
}

\author{
Nurul Atikah Binti Hamat ${ }^{1,2}$, Zulkiflee Salahuddin ${ }^{1}$, Rosdan Salim² \\ ${ }^{I}$ Department of Otorhinolaryngology, Hospital Raja Perempuan Zainab II, Kota Bharu, Kelantan, \\ Malaysia; ${ }^{2}$ Department of Otorhinolaryngology-Head and Neck, Universiti Sains Malaysia, Health \\ Campus, Kota Bharu, Kelantan, Malaysia
}

\begin{abstract}
Tick infestation in the ear canal may have variable clinical presentations. We present here a case of facial nerve paralysis in a 73 years old lady due to intra aural tick infestation. The patient presented with left otalgia, vertigo and left sided facial asymmetry. The case could be confused with cerebrovascular accident or transient ischemic attack.
\end{abstract}

IMC J Med Sci 2017; 11(1): 29-31

\section{Introduction}

Foreign body, either animate or inanimate, in the ear is a common clinical condition encountered in otorhinolaryngology (ORL) practice. Among the live foreign bodies, ticks are easily transmitted from domestic animals to human mainly through direct contact [1]. Intra aural ticks can cause otitis externa, tympanic membrane perforation and otitis media. A neglected tick bites can cause complications such as allergic reactions, infections and rarely may lead to facial paralysis [2]. Most common presentations include otalgia which is followed by bleeding, vertigo and tinnitus. However, cases of isolated facial nerve paralysis due to tick infestation in auditory canal are rare and are less commonly reported in the literature [17]. We present here a case of left facial nerve paralysis following tick infestation in ear canal in a 73 years old lady.

\section{Case report}

In July 2016, a 73 years old lady, with no known medical illness before, was admitted in medical ward with suspicion of transient ischemic attack (TIA). She experienced spinning sensation after waking up from sleep. The spinning sensation had a sudden onset that lasted for the whole day. It was aggravated by opening of the eyes. The symptoms were associated with nausea, vomiting and mild headache. On the same day, she developed left sided facial asymmetry and facial weakness (Figure 1). She was unable to close the left eye fully, with persistent drooling of saliva from the weakened left angle of the mouth. The hearing was reduced on left side. She gave history of left ear pain for one week prior to current presentation. However, there was no tinnitus, no ear bleeding or ear discharge. The speech was slurred but there was no blurring of vision. There was no weakness of the limbs. At emergency department, a computed tomography (CT) of brain suggested features that could represent foramen of Monroe haemorrhage, calcified lesion or vessel. Initially, she was managed by medical team. Subsequently, she was referred to otolaryngology (ORL) team as the case was not clinically suggestive of TIA. Upon examination, there was a left lower motor neuron facial nerve palsy grade IV House and Brackmann's Classification [8]. Patient also had horizontal nystagmus to the right. Cerebellar signs were normal. Upon otoscopic examination, a tick was found over posterior ear canal. It was already disengaged from the wall of the ear canal and we could easily remove it with suction. However, the species of the tick could not be determined. There was healed tympanic membrane on left side and on right side a small central perforation of tympanic

Address for Correspondence:

Dr. Nurul Atikah Binti Hamat, Medical Officer, Department of Otorhinolaryngology-Head and Neck, Universiti Sains Malaysia, Health Campus, 16150 Kota Bharu, Kelantan, Malaysia.Email: asadun7@gmail.com 
membrane was noticed. Rinne's test on right side was negative and left side was positive while Weber test was lateralized to right. Otoacoustic emission test was performed and refer bilaterally. Pure tone audiometry results showed right mild to severe mixed hearing loss and left side moderate to profound sensorineural hearing loss, while the tympanometry results were type B on right side and type A on left side.

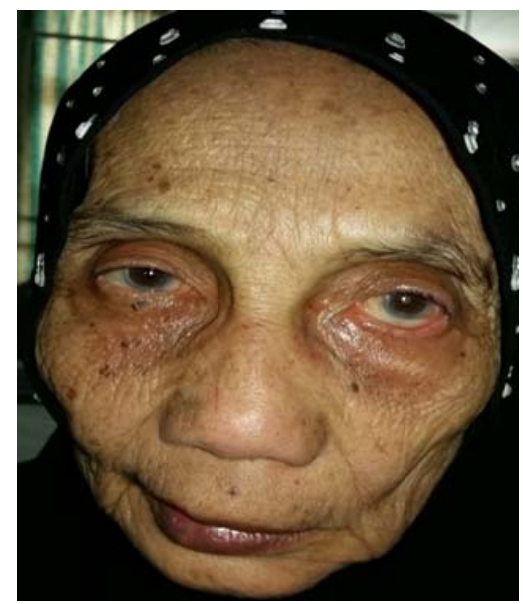

Fig.1: Left facial nerve paralysis grade IV House and Brackmann's Classification [8].

She was prescribed betahistine $24 \mathrm{mg}$, prednisolone $40 \mathrm{mg}$, methylcobalamine $500 \mathrm{mcg}$ and intravenous augmentin. Levofloxacine eardrop and artificial eyedrop were also administered. Her symptoms improved and she was discharge with the above medications. Repeated pure tone audiometry one week later showed improvement of her hearing on left ear to moderate sensorineural hearing loss and also reversal of her symptoms. The facial nerve palsy resolved completely 2 weeks later on follow up. Based on the above, it was concluded that facial nerve palsy was due to the tick infestation. Informed consent was obtained from the patient for the publication of the case.

\section{Discussion}

Tick infestations in the ear canal have been reported from all over the world particularly from tropical countries such as in India, Malaysia, Sri Lanka and Turkey [1-7]. It is a common occurrence seen in the east coast of Peninsular
Malaysia and usually encountered in the dry months but also can occur anytime of the year [5]. There are two main families of ticks that are of medical importance to human, namely Ixodidae (hard tick) and Argasidae (soft tick) [9]. Both hard tick and soft tick secrete saliva together with enzymes and anticoagulants from their salivary gland into the skin. Otalgia is likely to be caused by these enzymes secreted during their attachment in the ear canal. These enzymes are capable of causing inflammation and pain. Ticks which belong to family Ixodidae have been widely implicated in causing nerve paralysis. These ticks are capable of producing neurotoxins from their salivary gland during the feeding cycle $[9,10]$. Neurotoxins have been shown to interfere the depolarization and acetylcholine release mechanism in presynaptic nerve terminal and cause blockade of transmission at neuromuscular junction with resultant nerve paralysis [10]. The passage of neurotoxins commences on third day of infestation and peak on fifth to sixth day. The onset of clinical signs usually occurs five to seven days after attachment of ticks to the ear canal [11].

In the present case, the patient had complaint of otalgia one week prior to vertigo, reduced hearing and facial paralysis. Peripheral facial nerve palsy was diagnosed based on the clinical presentation weakness of all facial nerve branches, drooping of the brow, incomplete lid closure, drooping of the corner of mouth, impaired closure of the mouth and dry eye. The challenging part in intra-aural tick infestation was successful removal of the tick from ear canal. It is a very distressing experience to patients, especially children. Most of the times, the removal is made difficult by the swollen and narrowed canal from previous multiple attempts by inexperienced medical personnel with inadequate instruments [2,3]. It is always a wise decision to refer patients with insect or foreign body in the ear to the centre with the expertise and adequate instruments for ear examinations. Two approaches for removal of tick from ear canal have been described [4]. One is by application of a noxious stimulus to induce the tick to withdraw spontaneously and the second approached is by mechanical removal. Several reagents have been used intra aurally to remove tick from the ear canal with variable success. Spirit, olive oil, sodium bicarbonate, petroleum jelly and liquid paraffin are 
among various preparations used to facilitate tick removal with none of them proven to be superior to another. Cocaine (10\%) has been used by Baharudin and group [4]. They found that removal of the tick became easier by doing ear suction or by using forceps under microscopy following administration of cocaine. Cocaine weakens the tick and as a result the tick gets dislodged from the tympanic membrane or wall of the ear canal. Cocaine also helps to reduce the pain and it decongests the swollen ear canal. However, in uncooperative children, removal under general anaesthesia is safer and less traumatic.

Ticks are common living foreign body in the ears especially in tropical countries. One must consider intra aural ticks in patient presented with otalgia, vertigo and facial nerve palsy, and should look for hidden ticks within the ear canal. However, at the primary care level, the other prevalent causes of facial nerve palsy need to be considered such as systemic viral infections, trauma, surgery, diabetes, local infections, tumour, immunological disorders and drugs [11]. In case of suspected intra aural tick infestation, early referral to ORL clinic is required to remove the ticks and to avoid further complications.

\section{Conflict of interest: None.}

\section{References}

1. Somayaji KSG, Rajeshwari A. Human otoacariasis. Indian J Otolaryngol Head Neck Surg. 2007; 59(3): 237-239.

2. Indudharan R, Dharap AS, Ho TM. Intra-aural tick causing facial palsy. Lancet. 1996; 348: 613.
3. Lazim NA, Mohammad I, Daud MK, Salim R. The many faces of intra-aural tick clinical presentation. J Pak Med Stud. 2012; 3(1): 34-37.

4. Asha'ari ZA, Abdullah B, Hasan S, Sidek DS, Jusoh NM. Isolated facial palsy due to intraaural tick (ixodoidea) infestation. Arch Orofacial Sciences. 2007; 2: 51-53.

5. Shibghatullah AH, Abdullah MK, Pein CJ, Mohamad I. Acute labyrinthitis secondary to aural tick infestation. Southeast Asian J Trop Med Public Health. 2012; 43(4): 857-859.

6. Edussuriya BD, Weilgama DJ. Case reports: intra-aural tick infestations in humans in Sri Lanka. Trans R Soc Trop Med Hyg. 2003; 97: 412-3.

7. Gürbüz MK, ErdoğanM, Doğan N, Birdane L, Cingi C, Cingi E. Case report: isolated facial paralysis with a tick. Turkiye Parazitol Derg. 2010; 34(1): 61-64.

8. House JW, Brackmann DE. Facial nerve grading system. Otolaryngol Head Neck Surg. 1985; 93: 146-147.

9. Grattan-Smith PJ, Morris JG, Johnston HM, Yiannikas C, Malik R, Russel $\mathrm{R}$, et al. Clinical and neurophysiological features of tick paralysis. Brain. 1997; 120: 1975-1987.

10. Vedanarayanan V, Sorey WH, Subramony SH. Tick paralysis. Semin Neurol. 2004; 24(2): 181-184.

11. Finsterer J. Management of peripheral facial nerve palsy. Eur Arch Otorhinolaryngol. 2008; 265: 743-752. 